Abstract 265 Table 2 Results of chi-square tests comparing premature ovarian failure (POF) prevalence with different chemotherapy schedules. OR: odds ratio; $\mathrm{Cl}$ : confidence intervals; TaxAC-CMF: Anthracycline and taxane - cyclophosphamide, methotrexate, and fluorouracil; VAI/Al: vincristine, adriamycin and ifosphamide/adriamycin and ifosphamide; EPI-IFO: epirubicin and ifosphamide; ABVD: adriamycin, bleomycine, vinblastine and doxorubicin; CHOP: cyclophosphamide, doxorubicin, vincristine and prednisone; FEC: fluorouracil, epirubicine and cyclophosphamide; BEACOPP: bleomycin, etoposide, adriamycin, cyclophosphamide, vincristine, procarbazine and prednisone

\begin{tabular}{llll}
\hline Schedule & POF prevalence & OR $(95 \% \mathrm{Cl})$ & p-value \\
\hline Platinum-based & $8 / 24(33 \%)$ & $1.6(0.6-4)$ & $\mathrm{ns}$ \\
TaxAC-CMF & $7 / 18(39 \%)$ & $2.0(0.7-5.7)$ & $\mathrm{ns}$ \\
VAI/AI & $2 / 8(25 \%)$ & $1(0.1-5.1)$ & $\mathrm{ns}$ \\
EPI-IFO & $9 / 19(47 \%)$ & $3.1(1.1-8.2)$ & 0.02 \\
Capecitabine & $3 / 4(75 \%)$ & $9.5(0.9-94.4)$ & 0.04 \\
ABVD & $2 / 41(4.9 \%)$ & $0.1(0.02-0.4)$ & 0.0001 \\
CHOP & $8 / 13(62 \%)$ & $5.6(1.7-18.2)$ & 0.004 \\
FEC + weekly & $3 / 16(19 \%)$ & $0.6(0.1-2.4)$ & $\mathrm{ns}$ \\
paclitaxel & & & \\
Oncocarbide & $0 / 4(0 \%)$ & $0.7(0.6-0.8)$ & $\mathrm{ns}$ \\
Fludarabine & $1 / 3(33 \%)$ & $1.5(0.1-17.0)$ & $\mathrm{ns}$ \\
BEACOPP & $3 / 5(60 \%)$ & $4.7(0.7-29.3)$ & 0.09 \\
Temozolomide & $2 / 7(29 \%)$ & $1.2(0.2-6.4)$ & $\mathrm{ns}$ \\
Monoclonal antibody & $8 / 24(33 \%)$ & $1.6(0.6-4)$ & $\mathrm{ns}$ \\
Trabectedin & $1 / 5(20 \%)$ & $0.7(0.08-6.8)$ & $\mathrm{ns}$ \\
High-dose & $22 / 24(94 \%)$ & $63.6(14-289)$ & 0.0001 \\
\hline
\end{tabular}

at cancer diagnosis and with every change of treatment. This has a very important implication: the necessity to implement fertility preservation strategies when the risk increases.

\section{THE IMPACT OF A NATIONAL MULTIDISCIPLINARY TUMOR BOARD FOR CANCER DURING PREGNANCY}

${ }^{1,2} \mathrm{~J}$ Heimovaara*, ${ }^{3} \mathrm{IA}$ Boere, ${ }^{4} \mathrm{~J}$ De Haan, ${ }^{5,6} \mathrm{~K}$ Van Calsteren, ${ }^{1,2,7} \mathrm{~F}$ Amant, ${ }^{8} \mathrm{~L}$ Van Zuylen, ${ }^{2,7} \mathrm{C}$ Lok* ${ }^{*} \mathrm{KU}$ Leuven, Department of Oncology, Leuven, Belgium; ${ }^{2}$ The Netherlands Cancer Institute (NKI) - Antoni van Leeuwenhoek, Department of Gynaecology, Amsterdam, Netherlands; ${ }^{3}$ Erasmus MC Cancer Institute, Erasmus University Medical Center, Department of Medical Oncology, Rotterdam, Netherlands; ${ }^{4}$ Amsterdam University Medical Centers, Vrije Universiteit Amsterdam, Department of Obstetrics and Gynaecology, Amsterdam, Netherlands; ${ }^{5}$ University Hospital Leuven, Department of Obstetrics and Gynaecology, Leuven, Belgium; ${ }^{6} \mathrm{KU}$ Leuven, Department of Development and Regeneration, Leuven, Belgium; ${ }^{7}$ Antoni van Leeuwenhoek, Center for Gynaecological Oncology Amsterdam (CGOA), Amsterdam, Netherlands; ${ }^{8}$ Amsterdam University Medical Centers, Cancer Center Amsterdam, Vrije Universiteit Amsterdam, Department of Medical Oncology, Amsterdam, Netherlands

\subsection{6/ijgc-2021-ESGO.238}

Introduction/Background* Physicians encounter pregnant women with cancer only incidentally, leading to lack of expertise or confidence to inform and treat these patients according to up to date guidelines and expert opinions. In the Netherlands, a national multidisciplinary tumor board (MDT) for cancer and pregnancy was founded in 2012, and consists of 33 physicians from all over the country. Requests to discuss patients in the MDT were received by e-mail and a recommendation letter was generated based on available literature and expert opinion of the board members.

Methodology All requests and recommendations were gathered in a database and analyzed for tumor type, stage, gestational age and recommendation given. A survey was sent out to 93 physicians who requested recommendations of the MDT, containing questions regarding the their experiences with the MDT and the impact on treatment decisions.

Result(s)* From December 2012 to May 2021, 209 recommendations were formulated, mostly regarding pregnant

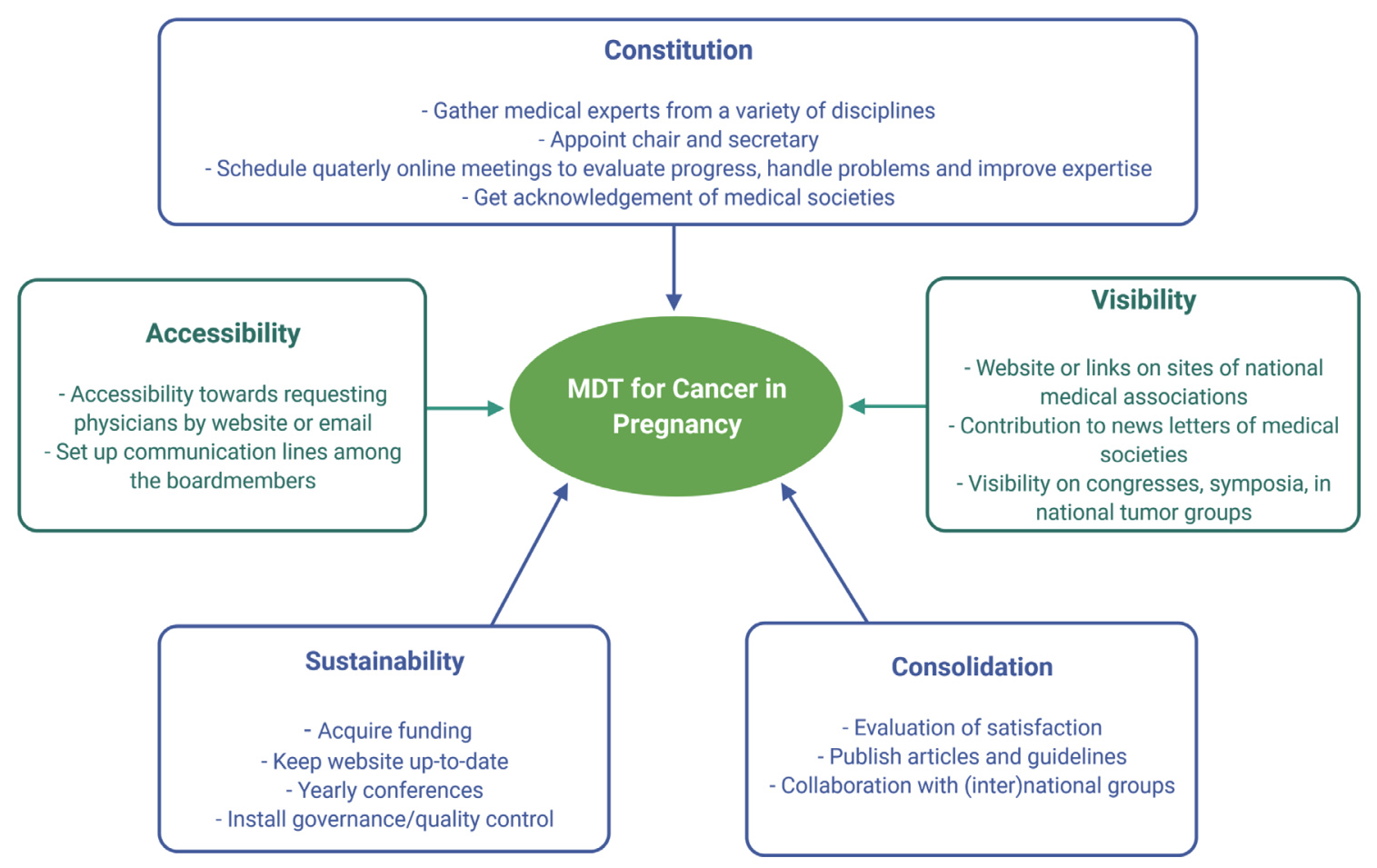

Abstract 290 Figure 1 
patients with breast cancer $(n=65)$, cervical cancer $(n=32)$, haematological malignancies $(n=32)$, rare cancer types $(n=30)$ and melanoma $(n=21)$. Questions were asked regarding possibilities of ionized imaging, chemotherapy, hormonal therapy or immunotherapy during pregnancy and possible effects on the child, the sequence of the different treatment components and questions on fertility. Response rate of the questionnaire was 54\%. Overall satisfaction with the recommendations of the MDT was high, and $94 \%$ of the respondents informed their patients about consulting the MDT and felt supported by the received recommendations.

Conclusion* A national MDT for cancer and pregnancy is frequently consulted and highly appreciated by physicians. Next to that, it increases expertise of its members about this rare coincidence of cancer and pregnancy. We highly recommend to establish an (inter)national MDT in each country. Figure 1 shows the important steps necessary to establish an MDT for cancer in pregnancy based on our experience.

\section{COMPARISON OF SERUM HE4 AND CA125 LEVELS IN THE EARLY POSTPARTUM PERIOD}

${ }^{1} \mathrm{R}$ Delic*, ${ }^{2} \mathrm{M}$ Stefanovic, ${ }^{3} \mathrm{~S}$ Krivec, ${ }^{1} \mathrm{~J}$ Koren. ${ }^{1}$ General and Teaching Hospital Celje, Department of Gynecology and Obstetrics, Celje, Slovenia; ${ }^{2}$ Sestre milosrdnice University Hospital Center, Department of Clinical Chemistry, Zagreb, Croatia; ${ }^{3}$ General and Teaching Hospital Celje, Department of Laboratory Medicine, Celje, Slovenia

\subsection{6/ijgc-2021-ESGO.239}

Introduction/Background* This study was undertaken to evaluate and compare CA125 and HE4 serum concentrations in the early postpartum period.

Methodology In a prospective designed, monocentric study (Department of Gynecology \& Obstetrics, General and Teaching Hospital, Celje, Slovenia, EU), 68 women who were in the 1-3rd day of postpartum period were included in the study.

HE4 and CA125 levels were obtained and analysed with regard to each day of postpartum period (1st, 2nd and 3rd day after delivery) as well as regarding the method of delivery (vaginal delivery; $\mathrm{n}=46$, elective (ElCS); $\mathrm{n}=15$ and emergency caesarean section (EmCS); $\mathrm{n}=8$ ).

CA125 and HE4 levels were measured in consideration of their reference intervals less than $35 \mathrm{IU} / \mathrm{ml}$ and less than 140 pmol/l, respectively (Elecsys CA 125 II $^{\circledR}$ assay and Elecsys HE4 ${ }^{\circledR}$ assay, Roche Diagnostics Ltd., Rotkreuz, Switzerland).

Data were analysed by ANOVA (MedCalc Software, Mariakerke, Belgium).

Result(s)* The CA-125 levels were frequently above the conventional cut-off limit of $35 \mathrm{IU} / \mathrm{mL}$; differences in mean concentrations between study groups were not found to be statistically significant (Group I, $\mathrm{n}=44$, 1st day after delivery, mean $=39,4 \mathrm{IU} / \mathrm{ml}$; Group II, $\mathrm{n}=19$, 2nd day after delivery, mean $=32,8 \mathrm{IU} / \mathrm{ml}$; Group III, $\mathrm{n}=5$, 3rd day after delivery, mean $=38,8 \mathrm{IU} / \mathrm{ml}, \mathrm{p}=0,708)$.

Regarding the method of delivery, women in the vaginal delivery group had seemingly higher levels of CA125 than the women in both caesarean section groups, but still not statistically significant $(44,0 \mathrm{IU} / \mathrm{ml}$ vs $29,7 \mathrm{IU} / \mathrm{ml}$ (ElCS) and 21,5 $\mathrm{IU} / \mathrm{ml}$ (EmCS), respectively; $\mathrm{p}=0,057$.

HE4 levels were within reference range and significant difference was also not observed between 1 st, 2 nd and 3 rd day postpartaly (Group I, n=44, 1st day after delivery, mean = $60,3 \mathrm{pmol} / \mathrm{l}$; Group II, $\mathrm{n}=19,2 \mathrm{nd}$ day after delivery, mean

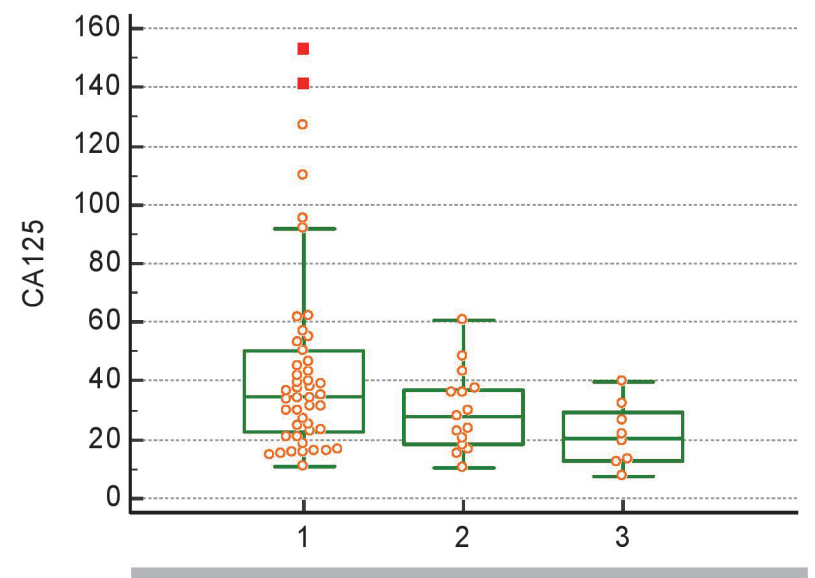

1-vaginal delivery 2- elective caesarean 3-emergency caesarean

Abstract 324 Figure 1

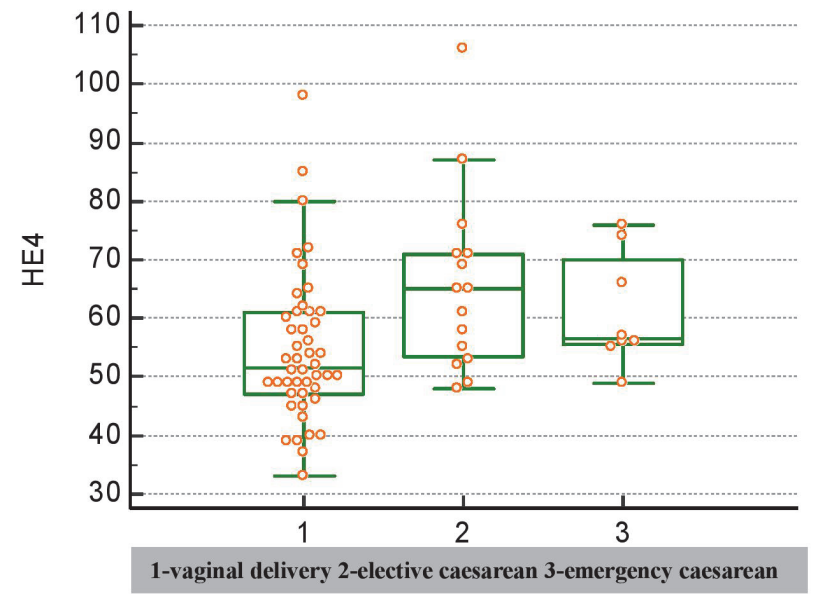

Abstract 324 Figure 2

$=54,3 \mathrm{pmol} / \mathrm{l}$; Group III, $\mathrm{n}=5$, 3rd day after delivery, mean $=49,2 \mathrm{pmol} / \mathrm{l}, \mathrm{p}=0,096)$.

Regarding the method of delivery, women in the vaginal delivery group had significantly lower levels of HE4 than the women in both caesarean section groups (54,5 pmol/l vs 65,7 $\mathrm{pmol} / \mathrm{l}$ (ElCS) and 61,1 pmol/l (EmCS), respectively; p=0,015. Conclusion* According to our preliminary results, HE4 is more reliable marker of malignancy during the early postpartum period than CA125.

\section{CONSERVATIVE SURGERY AND OVERALL SURVIVAL IN YOUNG WOMEN WITH A DIAGNOSIS OF BORDERLINE OVARIAN TUMOR}

M Laseca Modrego*, 0 Arencibia Sanchez, D González García-Cano, AF Rave Ramirez, A Martín Martínez. Maternal Child Hospital of the Canary Islands, Oncology Gynecology Department, Las Palmas de Gran Canaria, Spain

\subsection{6/jgc-2021-ESG0.240}

Introduction/Background* Radical surgery is the main treatment for Borderline Ovarian Tumors. Surgery includes hysterectomy, double adnexectomy, omentectomy and appendectomy if the histological line is mucinous. 